\title{
LA VOCALIZACION DE LAS SONANTES INDOEUROPEAS EN GRIEGO
}

This paper is a critical survey of recent work on vocalization of indoeuropean resonants after some authors having pointed at the deficiences of the traditional liypothesis on this issue. After examining the location of the anaptyctic vowel an effort is made to find the conditions in which the panlelenic vocalization in $u$ and $i$ took place. Concerning the $a / 0$ vocalization the views of Meillet, Morpurgo, Ruijgh, Müllestein, Georgiev, Adrados, O'Neil, Wyatt, Strunk, Bader and Moralejo are analyzed and a phonetical and phonological interpretation of the problem is proposed. Fin lly Heubeck's theory on conservation of * $r$ in Mycenaean is criticized following Adrados and Moralejo and adding new arguments: existence of $"$ vocalization in Mycenaean, unlikelihood of the relative chronology of this pirenomenon with regard to other phenomena, loss of ${ }^{*} \mathrm{H}_{2}$ in such sequences as ${ }^{*} r H_{1},{ }^{*} \mathrm{rH}_{2}$ and the treatment of the group $* r i$.

\section{INTRODUCCIÓN}

I.x. Después de haberse mantenido durante largo tiempo un esquema casi universalmente aceptado de los tratamientos en griego de las sonantes indoeuropeas ${ }^{1}$, en los últimos años, una serie de trabajos de gran importancia en calidad y cantidad han ido poniendo de relieve, descle diversos puntos de vista, las deficiencias de la opinión común sobre el tema, especialmente cuando se han añadido materiales nuevos a los ejemplos repetidamente citados antes.

Son muiltiples las dificultades suscitadas por el análisis de estos tratamientos y afectan a diversos campos de la lingüistica griega. En primer lugar podemos citar las que inciden en los estudios de dialectologia, como son, entre otras, las divergencias de los resultados en eolio, el problema de los tratamientos en arcado-chipriota y la posición del micévico. De otra parte es asunto debatido el de la cronologia de estos

1 Al hablar de sonantes en este artículo me refiero exclusivamente a ?. !, $m$, $n$. Los tratawientos de $i, n$ no son analizados aquf. 
cambios, desde la desconfianza que Risch ${ }^{1}$ maniliesta sobre la posibilidad de una datación, hasta la hipótesis de Heubeck ${ }^{2}$ quien cree que en micénico subsiste aún sin tratar la vibrante vocilica.

Pero lo que mais complica la cucstiún es que en ella como en pocas se produce un abierto conflicto entre la necesidad de colierencia, de regularidades, que el método lingüistico requiere y la profusión de escollos que a esta colierencia y regularidad parece oponer la multiplicidad de resultados que registra la vocalización de las sonantes. Resulta bastante difícil reducir a esquemas científicamente admisibles las diferentes posiciones y timbres de la vocal de apoyo e insertar a un tiempo estas cuestiones en las ya citadas de cronología y distribución dialectal de un modo colierente. No es extraño, por todo ello, que se haya llegado a posiciones divergentes, cuando no contradictorias, sobre el tema o a que se hayan silenciado algunos aspectos importantes del mismo.

La intención de este artículo es la de pasar revista a algunos trabajos recientes y, a partir de las valiosas aportaciones que en ellos se contienen, tatar de obtener un esquema completo de las vocalizaciones de las sonantes indoeuropeas en griego. Nos fijaremos para ello fundamentalmente en dos aspectos: la posición y timbre de las vocales de apoyo y la cronología relativa del cambio, con especial interés en el tema de la posible conservación de ${ }^{*} r$ en micénico. Asimismo trataremos de valorar la importancia que el estudio de estos resultados puede tener para la dialectología griega.

I.2. Antes de comenzar el estudio de los datos conviene hacer referencia a algunos problemas previos. Ya Ruijgh ${ }^{3}$ señalaba tres limitaciones básicas a la hora de analizar los testimonios griegos sobre este tema. La primera, la escasez de inscripciones, especialmente en lesbio, antes del 400 , años por los que comicnzan a notarse ya las influencias de la koiné ática o de la koiné occidental. La segunda, la alteración de los textos de la lírica lesbia, sometidos a hechos de koiné o a hipereolismos, que vienen a añadirse a los epicismos que ya presentaban Safo y Alceo. La tercera, por último, la influencia de la épica en los nombres de persona.

1 E. Risch, "Die Gliederung der griechischen Dialekte in neuer Sicht», Muserwm Helveticum 12, 1955, pp. 6r-76.

2 A. Heubeck, "Syllabic $r$ in Mycenaean Greck?n, Acta Mycenaca, Salamanca, 1972, II, pp. 55-79.

3 C. J. Ruijgh, sLe traitement des sonantes vo;elles dans les dialectes grecs et la position dy mycénienn, Mnemosyne 14, 196x, pp. 194-195. 
Otro escollo no fácil cle salvar es determinar en los timbres de las vocalizaciones cuándo hemos de interpretar una vocal como resultado de la vocalización de la sonante y cuándo se trata de una vocal originaria. l'or ejemplo, en el (aso del tiembre $o$, éste puede, en principio, proceder de la vocalización de la sonante o de un grado $o^{1}$. Algo parecido puede decirse ante la aparición de timbres $i, u$, que pueden proceder de $i, u$ indoeuropeas, según tendremos ocasión de examinar más adelante.

Ante todos estos problemas hay, en mi opinión, dos principios metodológicos fundamentales a seguir: de un lado, es preciso examinar la categoría morfológica a la que pertenece la palabra en cuestión y el grado vocálico que habitualmente presenta; y de otro, debe ampliarse lo más posible la base etimológica comparativa para evitar interpretaciones erróneas basadas en un par de casos que no se adaptan al resto de las formas que se relacionan con ellos.

r.3. Es sabido que es en el marco de los problemas silábicos donde se integran fundamentalmente los rasgos de comportamiento de las sonantes, fonemas que, como ya señalaron Saussure y Grammont ${ }^{2}$, deben a su abertura media la posibilidad de funcionar ya como parte cerrada (junto a fonemas más abiertos, las vocales), ya como parte abierta (junto a otros más cerrados, las consonantes) de la sílaba, posibilidades que se actualizan en virtud del juego de alternancias vocálicas. En las secuencias consonante más sonante más vocal (TRE) puede surgir entre la consonante y la sonante, en virtud de una pronunciación en tempo lento, un glide ( $T^{\circ} R E$ ) que puede luego fonologizarse o no ${ }^{3}$, con los resultados TERE o TRE, respectivamente. En cuanto a las sílabas cuyo centro era una sonante $(T R T)$, la tendencia general de las lenguas indoeuropeas ha sido convertirse en silabas fonológicamente normales mediante la pronunciación de estas secuencias con un apoyo vocálico $\left(T^{\circ} R T\right.$ o $\left.T R^{\circ} T\right)$ que acaba por convertirse en una vocal plena (TERT o TRET). Los problemas en el estudio de la vocalización de las sonantes indoeuropeas surgen a partir de este punto: en qué lugar aparece esta nueva vocal plena (que en el caso de * $\eta{ }^{*}{ }^{*} u$ acaba en griego

1 Cf. A. Morpurgo-Davies, "The Treatment of * $r$ and * $!$ in Mycenaean and Arcado-Cypriann, Atti e menorie del $r{ }^{\circ}$ Congresso Internazionale di Micenologia, II, Roma, r968, pp. 792-793.

2 M. Grammont, Traite de Phonétique, Paris, 1939, p. 98 y ss.

s Cf. F. R. Adrados, "La vocalización de las sonantes iudoeuropeas", ENIERITA 26, 1958, pp. 249-309, recogido en Estudios sobre las sonanles y laringales indoeuropeas, Madrid, 1973. 
por absorber a la sonante) y qué timbre adopta. Y precisamente a estos problemas vamos a aludir en los apartados siguientes.

I.4. No queremos entrar aquí a recoger y discutir las interpretaciones que sobre estos temas se han hecho en los estudios de indoeuropeo. Para ello el lector cuenta con dos excelentes estados de cuestión en los trabajos de Adrados y Gil ${ }^{2}$.

\section{LUGAR DE LA vocAL, DE APOyo}

2.I. Lil hecho es que en griego, como en otras lenguas indoeuropeas, aparece la nueva vocal antes de la sonante $\left({ }^{\circ} r>\alpha \rho\right)$ o después de la sonante $\left(r^{0}>p \alpha\right)$. La razón de ser de esta alternativa fue planteada ya por los neogramáticos, y se le dieron diversas interpretaciones. Mientras para Osthoff ${ }^{2} p \alpha$ era fonético cuando la palabra anterior terıniuaba en vocal, pero $\alpha p$ lo era cuando terminaba en consonante (esto es, serían

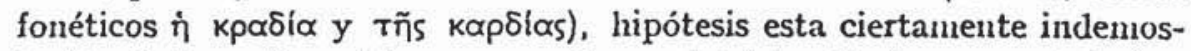
trable, Lejeune ${ }^{3}$ considera $\alpha \rho$ regular en inicial y final y $p \alpha$ en interior, aunque reconoce que pueda haber influencia de los grados plenos. Diferencias basadas en hechos de acento fueron postuladas por Kretschmer 4 , según el cual ${ }^{*} r>\rho \alpha$ pero si va acentuada secundariamente da $\alpha \rho$

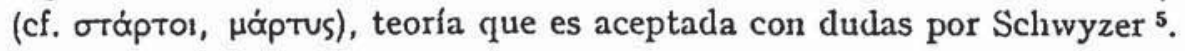
De otra parte, diferencias entre un "grado reducido" y un "grado cero" fueron invocadas por $\mathrm{Hirt}^{6}$, en lo que es seguido recientemente por Hoenigswald ${ }^{7}$. Iil propio Hirt $^{8}$ señala, no obstante, como posibiliclad la metátesis. Ante estas tentativas de explicación hay que poner de manifiesto que el análisis de los datos nos deniuestra la existencia de casos que contradicen estos esquemas, según tendremos ocasión de ver al examinar los materiales.

1 Adrados, ob. cil., J. Gil, ela apofonia ell indoeuropeo, EC 59, 1970, p. 4 y Ir.

2 H. Osthoff, Morphologische Unlersuchungen 2, Leipzig, 1879, pp. 144-145.

3 M. Lejeume, Phonétique historique du Mycénien el du Grec Ancien, Paris, 1972, pp. 196-197.

- P. Kretschwer, oIndogernanische Accent- und Lautstudiens, $K Z$ 31, 1892. p. 390 y ss.

- E. Schwyzer, Griechische Grammatik, Munich I, 1968, p. 342.

- H. Hirt, Indogermanische Grammatik, Heidelberg, II, 1921, p. 98 y ss.

7 H. M. Hoenigswald, Indo-Iranian Evidences, Evidence for Laryngeals (ed. W. Winter), La Haya, 1965, p. 97.

- H. Hirt, Kleiue grammuatische Beiträge», $I F$ 12, 1901, p. 232 y ss. 
Hay otra propuesta, que basa las diferencias de posición en diferencias de tensión articulatoria, debida a Grammont', seguido por Adra$\operatorname{dos}^{2}$ : $p \alpha$ refleja una tensión reciente en la articulación y $\alpha \rho$ una decreciente.

Por tiltimo, Kurylowicz ${ }^{3}$ recurre a la extensión morfológica como explicación: ya en indoeuropeo había tart por extensión morfológica del vocalismo $a$, mientras que $t_{r} t$ evolucionaba a trat. Este punto de vista es seguido, con variantes, por $\mathrm{O}^{\prime} \mathrm{Neil}^{4}$ sobre cuyas teorías tendremos ocasión de volver.

2.2. De estas vacilaciones se ha hablado fundamentalmente para las líquidas, ya que las nasales evolucionaron en griego normalmente a $\alpha$ (y a $\propto v$ más vocal o semivocal, donde no hay posibilidad de variación en la posición de la vocal de apoyo). Pero Osthoff ${ }^{5}$ postula un

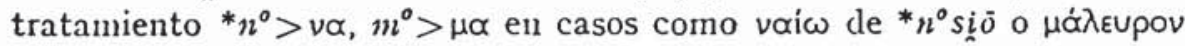
de ${ }^{*} m^{\circ}$ leuron, si no es contaminación del grado cero "regular" ă $\lambda \varepsilon v p o v$

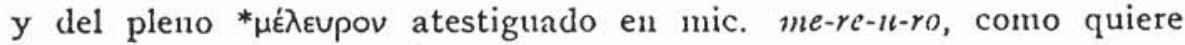
Ruijgh ${ }^{6}$.

2.3. In todo caso, dada la dificultad de obtener un esquema válido definitivainente para todos los casos, parece aceptable que ambas pronunciaciones con vocal de apoyo precedente y siguiente fueran variantes libres en indoeuropeo, situación que en griego deja huellas en casos lien conocidlos como kapтepós/kparepós, etc. Iin general, en las lenguas indeeuropeas se ha tendido a fijar una sola posición de la vocal de apoyo en cada lengua, o al menos en cada rai dentro de cada lengua, pero todo ello tiene el aspecto de ser una fijación secundaria, posterior a un estado de vacilación en la posición, según postula Adrados ${ }^{7}$. Lo que es claro es que existe influencia analógica de los grados plenos: hay $\sigma \tau \varepsilon \lambda-/$

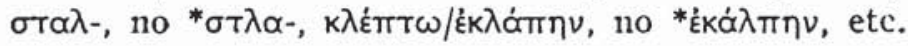

1 M. Crammont, ob, cil., p. 98 y ss.

2 F. 12 . Adraclos, Estudios..., p. 19.

3 J. Kurylowic\%, I'apophonie en indo-europeen, Wroclaw, 1056. p. 181 y ss.

- J. I. O'Neil, The Treatment of Vocalic $R$ and $L$ in Greek», Glolla 47, 1969, pl. $8-46$

- II. Osthoff, Morphologische Untersuchtungen 5, I,eipzig, 1890, p. III y ss.

- C. J. Ruijgh, Elabdes sur la grammaire et le vocabulaire du grec mycénien, Ansterdam, 1967, p. 176 .

7 I. R. Adrados, Estudios..., p. I9. 


\section{Trambe $"$}

3.I. Al iniciar el problema de los timbres de la vocalización de las sonantes quiero referirme, en primer lugar, a la posibilidad de la existencia panhelénica de los timbres $u$ e $i$ que voy a considerar con mayor detenimiento por tratarse de un punto especialmente conflictivo y muy eludido en la bibliografía reciente.

3.2. Desde antiguo se planteó ya el problema de algunos grados cero que presentaban vocalización $u$ junto a la sonante. A partir de algunos precedentes, Hirt ${ }^{1}$ propuso la existencia de una vocal reducida velar como origen de la $u$. Tal postulado, sin embargo, se enfrenta con dificultades evidentes. En primer lugar, deja sin explicar los tratamien-

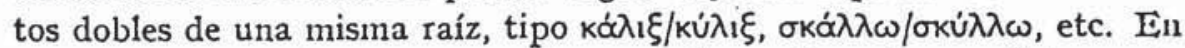
segundo lugar, Hirt se muestra incapaz de explicar las condiciones de aparición del grado reducido con vocal velar, del grado reducido con vocal palatal y del grado cero. Basar tales diferencias de grado solamente en la aparición de vocalizaciones diferentes y a la vez explicar las diferentes vocalizaciones por diferencias de grado es evidentemente un círculo vicioso. En tercer lugar, la crítica a mi entender más fundamental a estas diferenciaciones entre grado reducido y grado cero que otros autores postulan también, me parece la de Lelmmann ${ }^{2}$. Según este autor no hay, fonológicamente hablando, diferencias entre grado reducido y grado cero y sólo puede liablarse de una grado apofónico átono.

3.3. Por su parte, Güntert ${ }^{3}$, que opera con una sola vocal reducida a la que denomina Schiva sccundum, señala que el tratamiento $u$ puede ser fonético en contacto con labiales, labiovelares, velares, $u, w$, pero siempre que en la sílaba siguiente aparezca $i, y$. Ni que decir tiene que quedan fuera de esta explicación los casos en los que up, etc., aparecen distribución, ni se explica por qué hay $a$ en palabras como $\beta \dot{\alpha} \lambda \lambda \omega$, de * g*o li $\bar{i}$.

3.4. Como mera constatación del hecho, pero sin ninguna explicación del mismo, aparecen citadas las vocalizaciones con $u$ por Schwyzer ${ }^{4}$

1 H. Hirt, ob. cil., 11, 1921, 1. $76 \mathrm{y}$ ss.

2 W. Lehmann, Prolo-Indo-European Phonology, Austin, 1955, p. 19.

3 H. Güutert, Indogermanische Ablathtprobleme, listrasburgo, 1916, 1) 32 y ss., y $100 \mathrm{y}$ ss.

- E. Schwyzer, ob. cit., I, p. 35 r. 
y por Lejeune en una breve nota ${ }^{1}$. Pero la bibliografía reciente tampoco lia progresado mucho en este sentido: así, el tema aparece arrinconado en una nota del extenso artículo de Bader ${ }^{2}$, aunque la autora reconoce que es un tratamiento fonético, y no se dice ni una sola palabra de él en el artículo de O'Neil ya citado, por señalar dos de los más extensos.

3.5. İl autor que más se ha detenido en el tema ha sido Adrados ${ }^{3}$, aunque con posterioridad a sus trabajos podemos citar algun material nuevo debido a Van Brock ${ }^{4}$. En síntesis, las conclusiones de Adrados son las siguientes: a partir de su teoría de las vocales de apoyo, piensa que el apoyo vocálico que recibia la sonante, inicialmente no fonológico, podía verse influido en su timbre por los fonemas vecinos al fonologizarse. Así, las labiales y guturales condicionan el timbre $u$, sin que tenga que seguir $y, i$, como proponía Güntert. La coexistencia de los resultados $u$ con resultados $a$ en estas condiciones se debe a la tendencia alternativa de la sonante a vocalizar con el timbre más abierto. Tras ampliar el material de Schwyzer, hace asimismo Adrados una observación importante: el carácter marginal, fuera de las alternancias habituales, de las palabras que presentan estas vocalizaciones con timbre $u$.

3.6. Un análisis detenido de los materiales para la vocalización $u$ nos permitirá precisar más. Lo primero de todo, hay que descontar algunos casos en los que la vocalización $u$ de la sonante es solo aparente:

3.6.r. Un primer grupo de casos lo constituyen aquellos en los que $u$ se ha interpretado como vocalización de la sonante, pero un análisis más minucioso pone de manifiesto que se trata de una $u$ indoeuropea.

3.6.r.x. Es el caso de gr. $\gamma \lambda$ urús, que procede de $*$ dluk-, como se

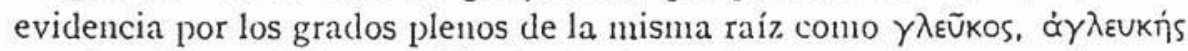
y mic. de-re-lt-ko5 . La dificultad planteada por la comparación con

1 M. L.ejemne, ob. cil., p. 197.

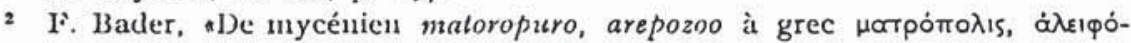
Bros: le traitement des sonantes-voyelles au premier millenairen, Minos 10, 19691970, pp. 7-63. La nota a la que aludo es la n. ${ }^{\circ}$ 129, p. 57.

3 F. R. Adrados, primero en el art. cit., luego en Studia Classica et Orientalia, enl 1959 y otros trabajos, recogidos en Eshudios...

- N. Van Brock, "De $\pi \cup \dot{\xi}$ à mäs», Melanges Chantraine, Paris, 1972, pp. 263276.

J. Claadwick, "Nycenaean Wine and the etymology of $\gamma \lambda$ ukús", Minos 9. 1968, pp. 192-197. 
lat. dulcis, que parecía proceder de *d!lk-se resuelve si pensainos que lat. dulcis deriva por metátesis de *dluk- debido a la inexistencia de palabras con *dl- en latín ${ }^{1}$.

3.6.I.2. Asimismo hay que ver una $u$ indoeuropea en $\gamma \lambda \dot{\varphi} \phi \omega$, ef. lat. $g l u ̈ b o$, de *gleubh-. La $\alpha$ de $\gamma \lambda \alpha \varphi$ upós procede de la disinilación de * $\gamma \lambda$ u

3.6.2. En otros casos, $u$ procede de la vocalización del apéndice de una laringal ${ }^{*} H^{*}$. Tal afirmación puecle constatarse cuando con las formas con $u$ alternan formas con vocal larga o palabras anatolias que

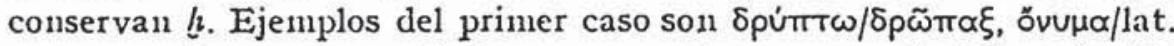

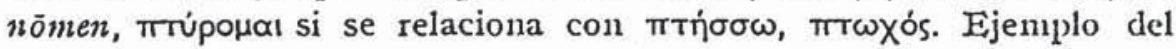
segundo caso es mũp frente a het. palılıur.

3.7. La mayoría del resto del material se deja dividir en una serie de grupos claros. El más evidente es el de las palabras en las que la sonante seguía a $\underset{\sim}{u}$ o a labiovelar.

3.7.I. Iǹn este apartado hay que tan sólo aludir, por bien conocido, al caso de $* t u^{\circ}>\sigma v$ - en míoupes $<{ }^{*} k^{* \circ} t u^{\circ}$ res, cf. lat. qualtuor ${ }^{3}$.

3.7.2. Asimismo se incluye en él la forma oúpł citada por el Etymo-

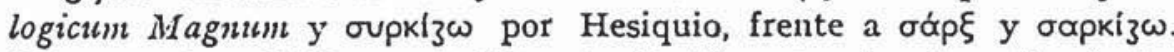
Para nuestro objetivo es indiferente decidir entre las dos etimologías propuestas para estas palabras: una que lo relaciona con la raíz indoeuropea *tuerk- 'cortar' (la evolución semántica tendría como paralelo

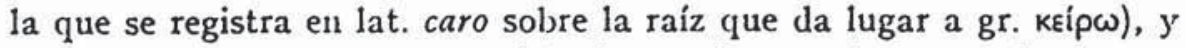
otra que lo compara a het. twekkas 'cuerpo'; en ambos casos la secuencia inicial era *tur-.

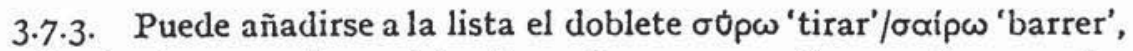
sin etimología segura fuera del griego. La comparación con el caso anterior impone una etimología a partir de ${ }^{*} t_{\mu}{ }^{\circ} \gamma$-.

1 Un paralelo podria ser lat. indulgeo si procede de la misma raiz que lict. daluki-, etc., cf. J. Pokorny, Indogermanisches Elymologisches Worterbuch, Berna, I959, I, p. 196 y ss. (obra que a partir de ahora citaremos sólo Pokorny).

2 Cf. P. Chantraime, Dictionnaire Etymologique de la Langue Grecque, Paris, I968, p. 227 (obra que a partir de ahora citaremos sólo Cliantraine).

- Cf. la solución sin vocal de apoyo y con la ufuncionando por tanto cono vocal en Tuptaios, si procede de "Túptos 'cuarto'; ef. otras soluciones en $\$$ 3.8.4. 
3.7.4. La alternancia entre $u$ y $\alpha$ sólo se explica si postulamos la siguiente evolución: antes de que en griego el grupo * $t_{1 l}$ - pasara a $s$-, surgió una vocal de apoyo entre $u$ y $r:{ }^{*} \iota_{n}{ }^{\circ} r$ - Sobre ella se ejercen entonces dos presiones diferentes: una, asimilatoria a la $u$ anterior (solución *tü $r$-); otra, normal en la vocal de apoyo cle la sonante, a adquirir el timbre más abierto, $a$ (solución ${ }^{*} t_{u}{ }^{a} r$-). Grosso modo podríamos hablar de una tendencia a la asimilación progresiva *uuur-, frente a una tendencia a la asimilación regresiva *tuar-.

Si se hubiese tratado el grupo *tu- antes de la vocalización de la sonante, el resultado habría sido ${ }^{*} s y^{*}$, con lo que la vocalización $u$ sería inexplicable.

Por el contrario, si $u$ fuera la mera vocalización de $u$, o mejor dicho, el alófono vocálico de este fonema, el resultado habría sido ${ }^{*}$ tur-, ya que no se registran palatalizaciones de $t$ ante $u$ fuera de casos analógicos como $\sigma u ́$ de $\sigma \dot{\varepsilon}$ y esta clase podría en principio invocarse para míoupes, pero la doble

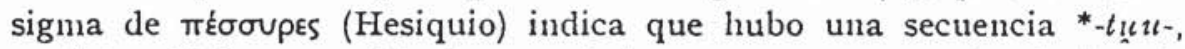

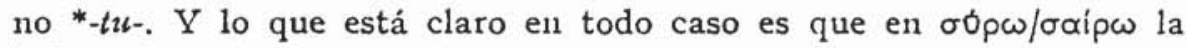
influencia analógica está descartada. Ambas palabras se separaron por el sentido y no hay huellas para postular *Túp$\omega$ en ningún dialecto.

3.7.5. En resumen, pueden señalarse tres estadios en la evolución,

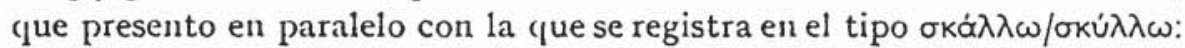

$$
\begin{aligned}
& \text { I: vocal de apoyo no fonológica *tu } r k \text { - } \quad * s k^{\circ} l \text { - } \\
& \text { II: doblete } a / u \quad{ }^{\prime \prime l u a r k-/ h u u r k-} \quad * \text { skal-/skul- }
\end{aligned}
$$

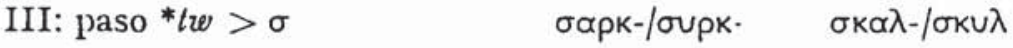

3.7.6. Paralelo, y si cabe, más claro aún, es el caso de yovń beoc. ßavớ. La vocal entre la labiovelar y $n$ puede sufrir la influencia asimilatoria del apéndice de la labiovelar, con "asimilación progresivan; ${ }^{*}{ }^{*} u n-$, o de la $n$, que tiende a vocalizar con el tiembre más abierto, $a:{ }^{*} g^{*} a n-$. A partir de esta situación, ambas formas evolucionan respectivamente a $\gamma u v-/ \beta \alpha v-$.

İn este caso el testimonio del micénico es de extraordinario interés. Hablo de la palabra derivada kut-na-ja. Sabemos que en micénico la secuencia labiovelar más consonante no se ha tratado aún, e.g.: qi-ri-ja-to ( $k^{\prime}$ rialo), qi-si-pe-e ( $k^{\prime}$ siphehe), qe-re-me-ne-u ( $k^{u}$ remneus), pu $u_{2}-k e-q i-r i$ (phurgek"ris), etc. Ello indica que ku-na-ja no refleja una palabra con labiovelar seguida de $n$, que se escribiría qa-na-ja. En cambio sabemos que ya ha ocurrido en época micénica la pérdida del apéndice de la 
laviovelar en contacto con $u$, e.g.: qo-tl-ko-ro (g"oukolos) $<*_{-}^{*} k^{*} o l o s$, $c-l l-k c-t o$ (eukhetoi $<^{*}-g^{\prime \prime} h c-$ ), etc. Para que $g^{*}$ se haya reducido a $g$ tiene que liaberse creado previamente $u$ de modo que * $g^{\mu \prime} n->g^{*} u n->$ gun-. La única razón de que ello suceda en kin-na-ja y no en qi-ri-ja-lo, qi-si-pc-he es que $u$ no es el resultaclo de la simple vocalización del apéndice de $g^{\sharp}$, sino que ha surgido como resultado de la vocal de apoyo entre $g^{*}$ y $u$, de tiembre $u$ (frente a la solución contraria que es seguida por beoc. $\beta \alpha \nu \alpha ́)$. No es ociuso observar que en griego alfabético, mien-

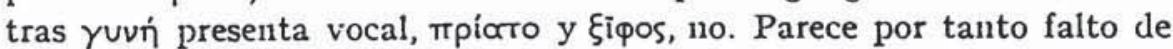

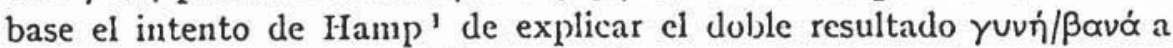

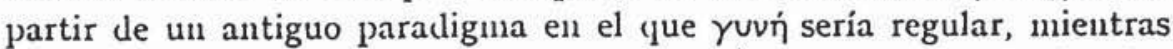
$\beta \propto v$ - procedería de casos oblicuos como el genitivo plural. Su explicación fracasa en cuanto que no puede dar cuenta fonética de resultados como $\xi \beta \propto \lambda \circ v$, etc., $y$, de otro lado, se ve obligado a postular en griego un

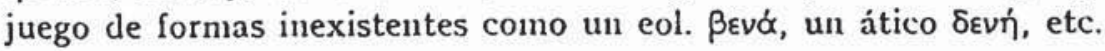

3.7.7. En un interesante artículo al que ya he aludido, Van Brock

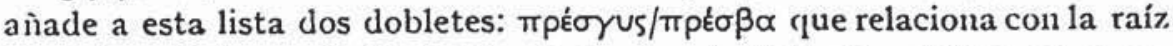

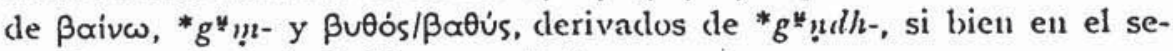
gundo caso la $\beta$ de $\beta$ uós es analógica.

3.8. Un segundo grupo de casos de vocalizaciones con $u$ lo constituyen aquellas palabras en las que había una labiovelar o $u$ que influye a distancia en el timbre de la vocal. Este fenómeno tiene paralelos bien conocidos como el retorromance leunga por lat. lingua, arm. awj, awcanel frente a lat. anguis, unguo. Se trata pues, de casos de asimilación a distancia. Como ejemplos de este grupo podemos citar los siguientes:

3.S.I. $\beta \lambda u ́ z \omega$ 'hervir', de ${ }^{*} g^{\prime} l^{0} d-$, de acuerdo con la glosa de Hesiquio $\beta \lambda u ́ \delta$ เov. úypóv, zéov, se relaciona con aaa. quellan 'hincharse', de la misma raíz asimismo que $\beta \alpha \dot{\alpha} \lambda \lambda \omega^{2}$. 'Tenemos por tanto un caso de asimilación a distancia, frente a la solución normal $a$, en dos palabras separadas por el sentido.

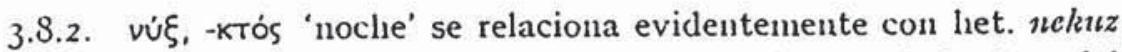
'al anochecer', derivados respectivamente del grado cero ${ }^{*} n^{\circ} g^{\sharp} h t$ y del pleno ${ }^{*} n c g^{\prime \prime} h$. Reconstruyo la raiz con ${ }^{*} g^{*} h$ (que evidentemente evolu-

1. L. I Iamp, eNotes on Liarly Greek Phonology", (ilolla 38, 1959-1900, pp. $200-203$.

2 Cf. Pokorny, pp. $47^{1-472 .}$ 
ciona a $k$ por asimilación a la $t$ siguiente) porque en las formas sin alar-

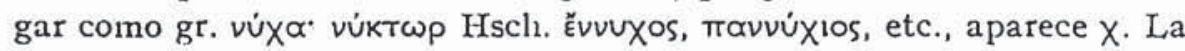
conservación del carácter velar aspirado en estas formas se debe, por fuerza, a una solución previa $u$ de la vocal de apoyo tras la cual la labiovelar pierde su apéndice: ${ }^{*} n^{0} g^{\underline{u}} l_{l-}>{ }^{*} n u g^{\underline{u}} l_{-}>v v X-$.

3.8.3. Lin una etimología que me parece irreprochable, Nadia van Brock ${ }^{1}$ interpreta $\pi \cup ́ \xi$ como procedente de ${ }^{*} p{ }^{n} k^{4} s$, grado cero de la raíz

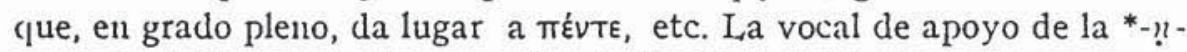
se vio influida, por tanto, por el apéndice de la labiovelar.

3.8.4. La palabra para el 'cuatro' en composición aparece en grado cero y pierde la labiovelar inicial: ${ }^{*} l^{*} l u r->{ }^{*} l_{u}(r-$. Ante consonante, surge una vocal de apoyo tras la ${ }^{*}-r:{ }^{*} \eta_{\imath} r^{\circ} \mathrm{C}$ - que, de acuerdo con lo dicho, puede verse influida durante el proceso de su fonologización por el timbre de $u ;{ }^{*} t_{u} u r u C$ - o bien adoptar el más abierto, $a:{ }^{*}$ tura $C$ - Ejemplo de la primera solución sería трuфá $\lambda \varepsilon ı \alpha$ y, fuera del griego, avést. čatru-, agal. petru-, lat. quadru-. Ejemplo de la segunda, тро́тrєз $\alpha^{2}$.

3.8.5. Es dudoso, si debemos situar en esta serie gr. yuuvós interpretándolo a partir de ${ }^{*} n^{o} g^{\sharp}$ mós (grado cero correspondiente al pleno que aparece en het. nekumanza), que evolucionará a *vuyuós, y cle ahí,

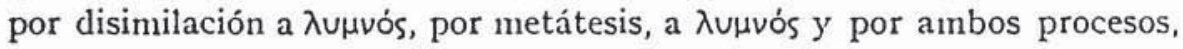
a ámo $\lambda_{\text {ú }}$ uarros. Otras soluciones son evidentemente posibles, como par-

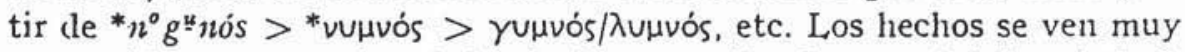
oscurecidos y no es desechable la intervención de un tabú lingüístico ${ }^{3}$, pero en todo caso es evidente que se trata de una raíz con labiovelar y que la $u$ procede de la fonologización de una vocal de apoyo, cuyo timbre se ha visto influido por el apéndice de la labiovelar contigua.

3.9. Un tercer grupo de casos lo constituyen una serie de palabras procedentes del substrato. No vamos a penetrar aquí en esta resbaladiza problemática, sino solamente a señalar que se ha atribuido por diversos autores a varios términos procedentes de un estadio pregriego un tratamiento con vocalización $u$ :

1 N. Van Brock, ob. cil.

2 Cf. \$ 3.7.I y la nota sobre Tuptaios, asi como mic. to-pe-za, sobre cuyo vocalismo $o$ volvercmos. No hay que descartar, con toda, la posibilidad de tomar la solución "truC como metátesis de * $\operatorname{turC}$ -

s Cf. Chantraine, p. $2 \downarrow^{2}$; Pokorny, p. 769 . Además, las soluciones con ō como aesl. nagu, lit. niogas hacen verosimil una raiz * nell" $3-g "$ "- 
3.9.I. Hay que citar en primer lugar la hipótesis de Georgiev ${ }^{1}$ que atribuye un tratamiento $u r, u l$ al Vorgricchischc indoeuropeo que postula en casos como gr. múpyos frente a gót. baurgs, naa. Burg ${ }^{2}$, o gr. Túußos frente a la solución con vocalismo $a$ Tópos, que deriva de * dhlublhos. Liste último ejemplo se la pucsto, nu obstante, en duda por motivos de sentido, ya que túpßos es el 'tumulo', mientras tó́pos es la 'fosa' 3 .

3.9.2. De otra parte, Heubeck ${ }^{4}$ reúne una serie de casos en los que explica un resultado $u r / r u$ en griego a partir de una ${ }^{*} r$ de origen anatolio, así por ejemplo múpyos junto a las glosas de Hesiquio фúpкos.

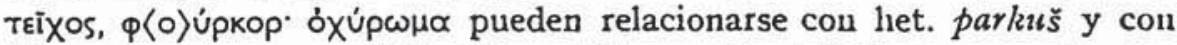

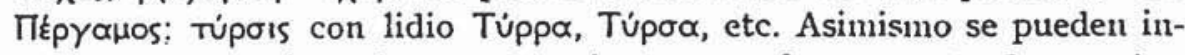
cluir en esta serie mpútavis, que, junto con foc. y cret. Bpuravev́w, - દiov, presenta la vacilación característica de los préstamos pregriegos, y que puede tener un correlato en hático puri 'señor's, y asimismo тúpavvos.

3.9.3. Aun hay una serie de palabras de origen dudoso que presentan $u$ junto a $l / r$ y que pueden aclararse en este contexto. Is el caso de $\beta \dot{u} p \sigma \alpha$, de $\tan$ discutida etinología ${ }^{6}$, en la que no podemos ni afirmar ni negar que la $u$ proceda de una sonante en grado cero, $\theta u \lambda \lambda i s /$ $\theta 0 \lambda \alpha$ kos, par cuyo carácter prehelénico se garantiza por el sufijo - $\alpha$ Kos y la alternancia $-v \lambda \lambda-\mid-\bar{v} \lambda-$ y que tiene correlatos con vocalismo $a$ como

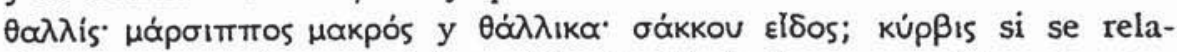
ciona con kapmós o con lat. corbis, etc. En todo caso, préstamos o no, se trata de un tratamiento griego y el griego ha hecho formar parte del sistema de su lengua a estos términos, por lo que no basta su condición de préstamos para excluirlas de un tratamiento $u$ griego de las sonantes.

1 V. Georgiev, Vorgriechische Sprachwissenschafl, Sofia, 1941, pp. 69-70.

2 La relación es ya de $P$. Kretschmer, "Nordische Lehnwörter in Altgriechischen, Glotta 22, 1933, p. 102. Cf. también D. A. Hester, 'P'Pelasgian', a new Indo-european Language? ", Lingua $\mathbf{1 3}_{3}, 1965$, p. 363 .

3 Cf. H. Iirisk, Griechisches Etymologisches Wörlerbuch, IIcidelberg, 19601972, II, p. 944 (a partir de ahora citaremos esta obra sólo l'risk).

- A. Heubeck, Praegraeca, Sprachliche Untersuchtsngen zum vorgriechischindogermanischen Substrat, Errlangen, 1961, p. $5^{8}$ y ss.

- Cf. J. Linderski, "Ettruskische 1Etymologien: zila9 und pur9», Glolla 40, 1962, p. 157 y ss.

- Cf. el restumen de hipótesis propuestas en Frisk, III, p. 57. 
3.ro. Un cuarto grupo dentro del tratamiento $\|$ lo constituyen una serie de formas de indole expresiva. Es bien sabido que el ámbito expresivo puede condicionar tratamientos fonéticos diferenciales, pero lo 'jue resulta en ocasiones difícil es determinar cuáles son estos ámbitos, es decir, qué térninos podemos catalogar como "expresivos». No obstante creo que podemos establecer sin grandes dudas una serie de apartados dentro de este grupo:

3.ro.I. Nombres de animales o sus actividades: $\beta$ púrixos, $\beta u ́ p \theta 0 r o s$

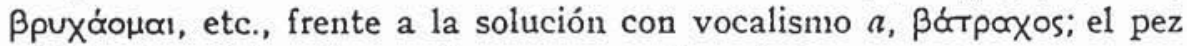

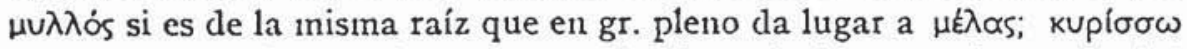
'topar', de la misma raíz que en grado pleno da lugar a képas y quizá $\mu u \dot{p} \mu \eta \xi$, palabra de difícil dilucidación y cuya relación con lat. formica, etcétera, no está definitivamente aclarada ${ }^{1}$.

3.I0.2. Defectos físicos: kuprós 'jorobado', con el mismo vocalismo que lat. curuus. Èn grado pleno esta raíz da lugar a una amplia serie

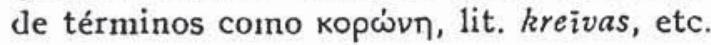

3.10.3. Hay otros casos en los que el vocalismo $u$ se combina con otros recursos reconocidamente expresivos, como la reduplicación, como

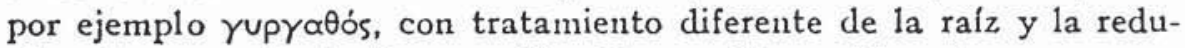
plicación a partir de ${ }^{*} \gamma u \rho-\gamma \alpha \rho \theta o ́ s$. Un vocalismo diferente aparece en

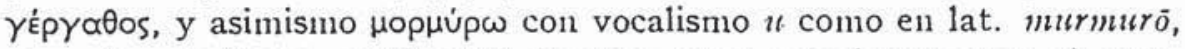
ai. mirmura 'fuego crepitante', frente a otras soluciones como ai. marmara- 'ruidoso', etc.

3.ro.4. Igualmente podemos hablar de un origen expresivo cuando en la palabra en cuestión se combina el vocalismo $u$ al recurso de la geminación, también expresiva. Es el caso de la serie de $\sigma \kappa u ́ \lambda \lambda \alpha, \sigma \kappa u ́ \lambda \lambda$, $\kappa \dot{ } \lambda \lambda \lambda \alpha, k v \lambda \lambda \delta_{s}$, etc.

3.10.5. Lin otros casos aún la expresividad es más dudosa, aunque

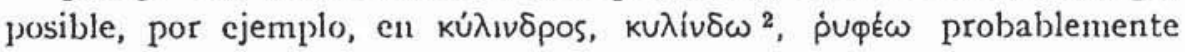
onomatopéyico, derivado de un grado cero *sybl - (cf. lit. surbiu, lat. sorbeo, etc.), que en grado pleno aparece en alb. gjerp-, de *serbh- y

1 Cf. Frisk, II, p. 272.

2 Si no procede de ${ }^{*} k^{* o} l$, como quiere J. Taillardat, Le groupe familier grec:

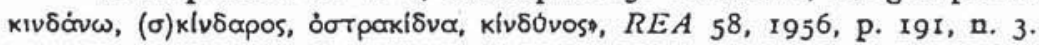


ell lit. srcbiu, de *srébli-, cf. gr. ṕopéw. Lìn algunos casos parecen coc-

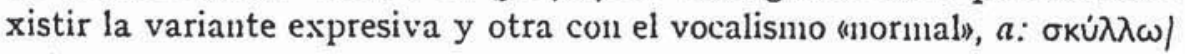
$\sigma \kappa \alpha \dot{\lambda} \lambda \omega \omega$, orup $\theta i z \omega / \sigma \pi \alpha i p \omega$, etc.

3.II. Descontados todos estos casos, nos queda aún una serie relativamente larga de palabras cuyos denominadores comunes son: en el aspecto fonético, vecindad de labial o gutural, que facilita la realización de la vocal de apoyo con timbre $u$; en el aspecto morfológico, su aparición fuera de series de alternancias moríológicamente normales; en el aspecto semántico, su aislamiento de grandes grupos semánticos, - bien el aprovechamiento de la vacilación en las vocalizaciones para marcar diferencias lexicales (lo que desde luego ocurre también en algunos grupos de los anteriormente analizados). La forma con vocalización $a$ se integra normalmente en series más amplias. Llegados a este punto, los condicionamientos últimos se nos escapan, y la razón por la que en unos casos se imponga lo que hemos venido llamando masimilación progresiva" $\left(k^{\circ} r>k \alpha \rho\right)$ y en otros la uregresiva" $\left(k^{\circ} r>k U \rho\right)^{1}$ como en $\beta a v \measuredangle / \gamma u v \eta ́$, no nos es accesible. Nos limitamos pues a citar algunos de estos casos no clasificables, con las características señaladas:

3.ri.r. Derivadas del grado cero de la raíz que lay en óyeipw y óyopó son ắyupıs y óyúprns, con vocalización $u$, que alterna con el

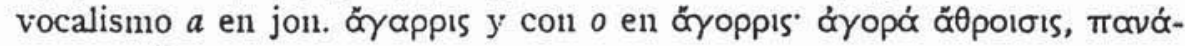
ropois, etc.

3.ri.2. El adverbio v̇móßpuxa (ya documentado en Homero), así como imoßpúxios (en los Himnos Homéricos), etc., presuponen un nombre raíz ${ }^{*} \beta p u ́ \xi$ que presentaria, en grado cero, la misma raíz que $\beta$ péx $\chi \omega$.

3.11.3. Litimológicamente, kú $\lambda_{1} \xi$ y $k \alpha \dot{\alpha} \lambda_{1} \xi$ son dos variantes disociadas por el sentido de la misma raíz.

3.Ir.4. En $\mu$ Ú $\lambda \eta$ tenemos el grado cero con vocalismo $u$ de la raíz *mel-, abundantemente representada en indoeuropeo ${ }^{2}$. No es seguro

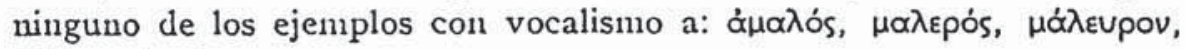
$\dot{\alpha}^{\prime} \lambda \dot{\varepsilon}^{\circ} \mu \mathrm{al}^{3}$, etc.

1 Naturalmente en el caso de la secuencia sonante más cousonante, la terminologia seria la contraria, regresiva, 16 , progresiva, $a$.

? Pokorny, p. 716 y ss.

3 Sobre este último, cf. J. Chadwick y L. Baumbach, The Mycenaean Greek Vocabulary*, Glolla 4I, 1963 , p. I70. 
3.Ir.5. Ianto $\sigma \varphi \tilde{p} \rho \alpha$ como $\sigma \phi \alpha i ̄ p \alpha$ represcntan soluciones de una raíz en grado cero con sufijo ${ }^{*}-i(e) H_{2}:{ }^{*} s p(h)^{o} r-i H_{2}{ }^{1}$, una con vocalismo $u$, otra con vocalismo $a$, fijadas antes del tratamiento del grupo $r+y^{\prime}$ (cf. con ü, oqupóv).

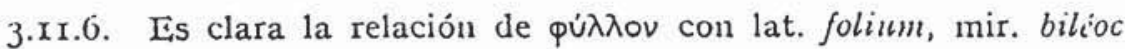
$(<*$ bhelio- $)$, etc., y probablemente pertenece a la misma raíz que da

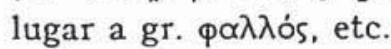

\section{TIMBRE $i$}

4.1. También se intentó desde antiguo dar respuesta al problema de la aparición de grados cero con vocal $i$ junto a la sonante. Hirt ${ }^{2}$ recurrió para ello a otra vocal reducida, ésta palatal, solución que se presta a críticas de la misma índole que la vocal reducida velar que invocaba para explicar la $u$ : no se aclara las alternancias del tipo $\sigma$ kaip $\omega$ Jokiptów ni puede dar cuenta de las condiciones de aparición de la tal "vocal reducida palatal». Por su parte Güntert ${ }^{3}$ recurría en unos casos

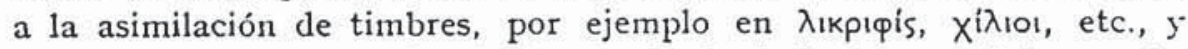

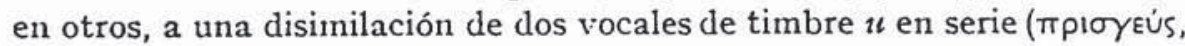
etcétera), explicación que no es aplicable a todo el resto de los ejemplos de la vocalización $i$. En cuanto a la serie de verbos con sufijo nasal

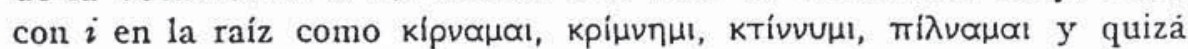
*ipvup según el epíteto de Zeus, ipvútios, Kuryłowicz ${ }^{4}$ los considera analógicos de un presente reduplicado, óvivnu reinterpretado como presente en $-\nu \eta$. La existencia de estas vocalizaciones pasa a pequeños apartados en los manuales de Schwyzer ${ }^{5}$ y Lejeune ${ }^{6}$. Adrados ${ }^{7}$ precisa que la vocalización $i$ puede verse fonéticamente determinada por una dental o por una $l$ alveolar contigua.

4.2. También en este caso caben precisiones, aunque los materiales para la vocalización $i$ sean mucho más problemáticos. Como en el caso

1 Sobre la alternancia $p / p h$ cf. recientemente F. Villar, AEl problema de las sordas aspiradas indo-europeas", RSEL I, I97I, pP. I29-160. La raiz aparece ampliamente documentada en indoeuropeo, cf. Pokorny, p. 992 y ss.

2 H. Hirt, ob. cil., II, p. 76 y ss.

3 H. Güntert, ob. cit., pp. 22-24.

- J. Kuryłowicz, ob. cit., p. 259 y ss.

s E. Schwyzer, ob. cit., p. 352.

- M. Lejeune, ob. cit., p. 208.

7 I. R. Adrados, Esludios.... p. $4^{\text {t. }}$ 
de la $u$, hay que descontar previamente aquellas palabras en las que la $i$, que pretendidamente procede de la vocalización de la sonante, se debe en realidad a otro origen.

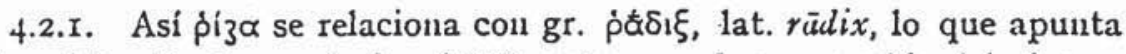
a la existencia de una laringal ${ }^{*} H_{2}$ entre $r$ y $d$, responsable del alargamiento y timbre de la $a$. Ello hace verosímil que dicha laringal sea palatal y que el timbre $i$ se deba a la vocalización de su apéndice: ${ }^{*} u_{\mathrm{C}} \mathrm{Hi}_{2}{ }^{\circ} \mathrm{di} \mathrm{H}_{2}$; cf. ya en micénico wi-ri-za. Hay en cambio $a$ en ṕós $\alpha \mu v o s$, con vocal de

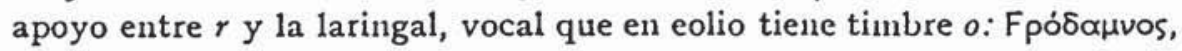

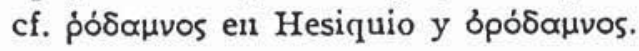

4.2.2. El caso de víoroua es dudoso. Si se piensa que se trata de ${ }^{*} n i-n s-0-m a i$, esto es, de un presente reduplicado y en grado cero, la $i$ sería la vocal normal de la reduplicación, para ello presenta el grave inconveniente fonético de que se esperaría vivoua. Si en cambio se piensa en un desiderativo con grado vocálico reducido: ${ }^{*} t^{\circ} s$-so-mai, $i$ procede fonéticamente de una vocal de apoyo con timbre $i$ ante $s^{1}$. La cuestión permanece abierta.

4.3. Descontados estos casos, el resto del material puede clasificarse en tres grupos.

4.3.I. Un primer grupo to constituyen las palabras en las que la sonante estaba palatalizada. Así, de oikтpós se crea el presente con $k$

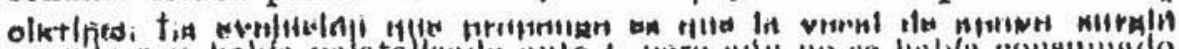

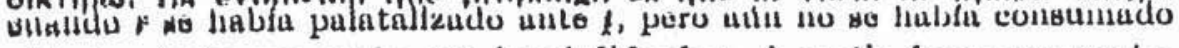
el tratamiento que acaba con la pérdida de $i$. A partir de ese momento, el tratamiento se consuma y los resultados son lesbio olkrippw, át.

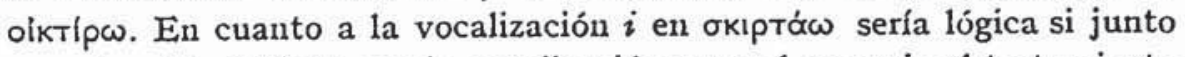

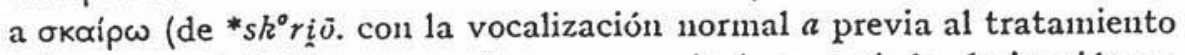

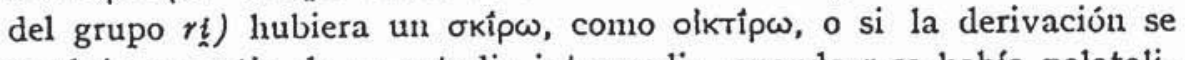
produjo a partir de un estadio intermedio, cuando $r$ se había palatalizado. Esta sería también la explicación de kı $\lambda \lambda$ ós y $k i \lambda \lambda$ os si proceden

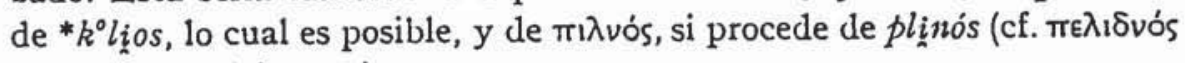

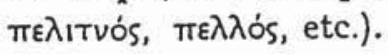

4.3.2. Otro grupo coherente lo constituye la serie de verbos con sufijo nasal, de acuerdo con la explicación antes mencionada de Kuryło-

1 Cf. bibliografla en lirisk, II, p. 305. 
wicz, con la salvedad de decir que en todo caso no se trata de analogías a posteriori (esto es, de la sustitución de las formas con vocalización normal por las con $i$ ), sino de una presión analógica ejercida en el proceso de fonologización de la vocal de apoyo.

4.3.3. Quedan, como en el caso de la vocalización $u$, un puñado de palabras de explicación dudosa: $k ı \gamma k \lambda i s$, quizá de una forma reduplicada sobre la raíz de $k \lambda l v \omega:{ }^{*} k i-k l i n-$, con $i$ en la reduplicación y disimilación; $\lambda ı$ ıкıрis, en donde puede haber asimilación vocálica, etc.

4.4. Aunque la vocalización $i$ no alcanza la extensión de la vocalización $u$, siguen subsistiendo las mismas dificultades que en ésta para determinar los motivos últimos de su aparición y por qué se impone a la vocalización $a$ que se produce también en casos en los que la sonante

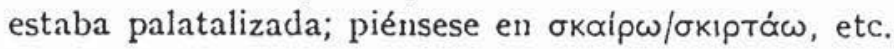

\section{Vocalización $e$}

5. La vocalización $e$ tiene unos limites muy claros ${ }^{1}$. Se limita al lesbio, en secuencias $r i$ - en las que la $i$ se consonantizó, y el grupo $r i$ se resolvió en una geminada palatal secundaria que provocó el timbre $e$ de la vocal de apoyo surgida ante ella, precisamente por su carácter pala-

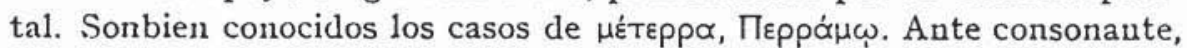
la geminada se redujo, por ejemplo, en тв́pтov. El fenómeno puede verse también en el eolio de Asia en casos como 'Iképtins. TÉppeos.

\section{Vocalización a/o. Opinión cominn y críticas a la misma}

6.1. Más interesante es sin duda el estudio de las vocalizaciones $a$ y $o$ porque en él se mezclan cuestiones de dialectología. La opinión común, largo tiempo sustentada y que llega, por ejemplo, a la fonética de Lejeune ${ }^{2}$ es la siguiente: las liquidas dan $\alpha \rho / p \alpha$, etc., en jónico ático y en clorio, pero op/po, etc., en eolio y arcado-chipriota. Las nasales dan $a$ en todo el griego. Los hechos no permiten, sin embargo, su reduc-

\footnotetext{
1 Es evidente que resultaria imposible hallar ejemplos claros de esta vocalización fuera de los que citaremos, dado que no pueden distinguirse de un grado $e$. I’or ello es metodológicamente más aceptable no tomar en consideración uma hipotética vocalización e del grado cero, más que en los casos que siguen.

2 M. L.ejeunc, ob. cil., p. 195 y ss.
} 
ción a un esquenı tan simple, por lo que no faltaron, descle bien pronto y desde frentes distintos, puntos de vista divergentes del mismo. Creo que merece la pena hacer una breve relación de las principales aportaciones en estc terreno.

6.2. Ya Meillet en $\mathrm{I}_{\mathrm{g}} \mathrm{O}^{1}$ señaló la posibilidad de 0 panhelética procedente de nasal sonante en algunos ejemplos como єйkoбı, öтотроร, Évтó leur répartition... son aussi indéterminables que celles qui règlent la répartition de up, pu et de $\alpha \rho, \rho \alpha$, sur le principe de laquelle on ne sait rien". La voz de Meillet resultó una auténtica predicación en desierto. Habrfamos de esperar hasta $195 \mathrm{r}$, en el que surge un nuevo detonante contra la opinión común, con el desciframiento del micénico.

6.3. Los ejemplos del micénico presentaban para $r$ resultados or/ro (al menos así se interpretaron en un principio las grafías con signos silábicos tipo to frente al tipo (o-ro) y algún ejemplo de $a r, r a: p a-w c-a$ |phárweha|, ka-po |karpós|, e-ra-pe-me-na |er(r)apménä|. Para las nasales había vacilación entre $o$ y $a$ incluso dentro de la misma palabra: pe-mo/ pe-ma, a-re-po/a-re-pa-, etc.

6.4. Para explicar esta alternancia se propusieron soluciones de diferentes indoles:

6.4.I. Una base fonética para estas distribuciones trató de buscarla Anna Morpurgo ${ }^{2}$, quien postuló para la nasal sonante que el timbre $o$ se debe a la vecindad de labial. En cuanto a las líquidas, concluye en otro trabajo ${ }^{3}$, que el resultado con $o$ se produce tras $u$. Desmienten, no obstante, una interpretación tan esquemática de los hechos los casos de pe-ma|pe-mo, to-pe-za, ma-to-pu-ro y un largo etcétera.

6.4.2. Por su parte Ruijgh ${ }^{4}$ piensa en una nivelación morfológica a partir de la solución -or/-a- en los heteróclitos, en dos flexiones: una $-o r$, otos y otra -ar, -atos. No obstante tal solución deja sin explicar el vocalismo de formas como e-ra-po, ri-me-ne, e-ra-pe-me-na, ka-po, etc.

1 A. Meillet, "Sur une origine de grec o*, MSL 16, 1910, pp. 217-220.

2 A. Morpurgo, s'esito delle nasali sonanti in miceneon, RAL 15, 1960, pp. $321-336$.

3 A. Morpurgo, art. cit. ell Alli Roma.

- C. J. Ruijgl, art. cit. 
6.4.3. Otra tentativa de explicación es considerar la posibilidad de que el micénico incluyera dos dialectos diferentes. Iniciada por Mühlestein $^{1}$, fue recogida con variantes en diversas propuestas por Georgiev ${ }^{2}$, y asimismo reflejada en la distinción sugerida por $\mathrm{Risch}^{3}$ entre un micénico normal y un micénico especial.

6.5. Por otra parte, la frecuencia de la vocalización o en micénico llevó a una serie de estudiosos a la conclusión de que, dado que ese tratamiento caracterizaba al eolio y al arcado-chipriota por oposición al jónico-ático y al griego occidental, el micénico se alineaba con el primer grupo. Ahora bien, no voy a insistir en el problema de la agrupación dialectal originaria, dado que es cuestionable que la isoglosa del tratamiento de las sonantes sirva para establecer una diferenciación dialectal antigua. Efectivamente, en una serie de trabajos ello se ińa poniendo en evidencia en mayor o menor medida, como veremos a continuación.

6.6. Adrados fue el primero en señalar ${ }^{4}$ la poca fiabilidad del tratamiento a/o como isoglosa diferenciadora de grupos dialectales. Respecto a las líquidas, hay o supuestamente eolia en todo el griego en

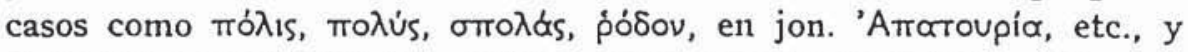
es forzado considerar una palabra como ßpotós un eolismo. De otro lado hay $a$ en arcado-chipriota y en eolio en palabras como orparós,

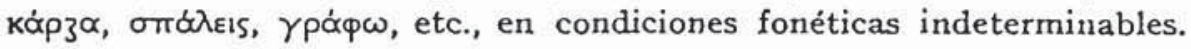
En cuanto a las nasales, Adrados se hace eco de Meillet con respecto al tratamiento panhelénico $o, y$ añade la presencia de $o$ fuera del eolio

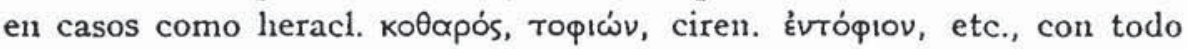
lo cual la distribución $o$ en eolio y arcado-chipriota, $a$ en el resto tiene todo el aspecto de ser una regularización tardía, que sólo pudo producirse a partir de un estadio previo de vacilación. Observa asimismo la posibilidad de un influjo fonético en los tratannientos: $o$ aparece fundamen-

1 H. Mühlestein, "Zur mykenischen Schrift. Die Zeichen za, ze, zor, $M H{ }_{12}$, 1955. p. I 21 n. 19 y p. 125 11. 40.

2 Se refiere concretamente al tema que nos ocupa en "Le traitement des sonantes voyelles indoeuropéennes et le problème du caractère de la langue mycenienne*, Acta Micenaea, Salamanca, 1972, pp. 361-379.

a E. Risch, "Les différences dialectales claus le Mycénien", Proc. of the Cambridge Colloguitm on Mycenaean Studies (ed. L. R. Palmer y J. Chadwick), Cambriclge, 1966, pp. 150-157.

* Primero en La dialectología griega como fuente para el estudio de las migraciones indoeuropeas en Grecia, Salamanca, 1952, luego en el art. cit. recogido en Esludios... 
talmente en vecindad de labial o gutural y el timbre puede verse influido por ellas. También es influible por una vocal $"$ siguiendo a la sonante. Con todo, el trabajo de Adrados vuelve a correr la suerte del de Meillet. Es más, curiosamente en una serie de estudios recientes se llega, sin citarlo, a puntos de vista semejantes o no discordantes con el mismo, como veremos.

6.7. Un nuevo golpe a la visión tradicional lo asesta Anna Morpurgo $^{1}$ al seũalar que la opinión común de un trataniento or/ro en arcado-chipriota se debe a un artículo de Fraenkel en I9Ir y se basa en pocos ejemplos, frente a los más abundantes de ar/ra. Ė̀n su trabajo demuestra de modo convincente que los casos con ar/ra no son préstamos, sino que este trataniento es fonético en ambos dialectos. No obstante, incapaz de admitir un doble tratamiento, desecha el carácter fonético de or/ro salvo tras $\underline{u}$, donde puede serlo, viéndose así obligada a admitir grados $o$, falsas etimologías, préstamos, etc., para los casos con vocal $o$.

6.8. En cuanto a $\mathrm{O}^{\prime} \mathrm{Neil}^{2}$ se ocupa del tema de $r$ y $l$ en griego y señala que ante vocal, ante $i, u$, en posición inicial y final, así como ante ${ }^{*}-k s$ (evolucionada a $\xi \circ$ a $\chi$ - ante el sufijo $-\mu \alpha$ ), la vocalización $a$ es panhelénica, con lo que en lesbio son fonéticos resultados como los producidos en $\chi \propto \lambda \alpha_{\alpha} \sigma \sigma o \mu \varepsilon v, \chi^{\alpha} \alpha 15$, etc. En el resto de los casos, ra, la son los resultados del jonio, la mayoría del eolio y el dorio, mientras or es el propio del lineal B y del arcado-chipriota. En eolio de Asia, lo corriente es $a$, aunque hay casos de $o$. En lesbio hay $a$ (áß $\beta \alpha \dot{\beta} \beta \eta$, órрó́kтఢ, $\beta$ pó́ke $(\alpha)$, etc.) pero el tratamiento $o$ es más normal e incluso al confundirse con los grados $o$ produce una extensión de los grados $o$ fuera del contexto de las prinitivas sonantes. Incapaz de admitir una alternancia a/o sobre bases fonéticas, concluye que el vocalismo o tiene su origen en algún subdialecto del eolio anterior a la migración, aunque reconoce que no es posible determinar el área en la que este dialecto pudo hablarse.

6.9. Desde otro frente contradice la distribución tradicional de los tratamientos $\mathrm{Wyatt}^{3}$, quien ante la existencia de $o$ en jónico-ático

1 En el trabajo ya citado de Alti Roma.

3 En el extenso artículo ya citado.

3 En un trabajo hiperueogramático: eSonant $/ R /$ and greek dialectology", S.HEA 13, 1971, pp. 106-1 22. 


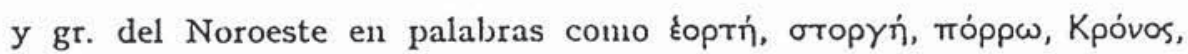
xpóvos y de $a$ en eolio (Safo, Alceo e Inscripciones )en palabras como

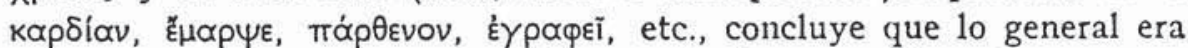
$\alpha \rho / \rho \propto$ pero hay casos de $o$ debidos a analogía con grados plenos con $\bar{o}$

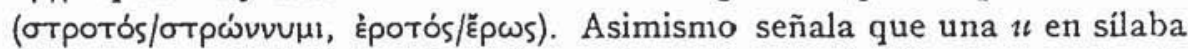
siguiente puede influir sobre el vocalismo (lo que había sido puesto de manifiesto antes por Adrados, a quien Wyatt no cita) ${ }^{1}$, en casos como

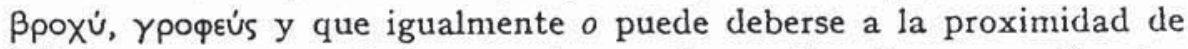
labial en casos como $\delta p \delta \mu \omega \mu \varepsilon v$, etc., por lo que ßpotós no es eolio, sino pregriego (ambas observaciones hechas asimismo por Adrados). Las reglas narcadas por Wyatt son:

a) Pregriego ${ }^{*} r>\alpha \rho / \rho \alpha$.

b) Protogriego $\alpha p / p \alpha>o p / p o$ ante sílaba siguiente con $u$.

c) En gr. del sur del Peloponeso, $\alpha>0$ ante la secuencia $p+$ cons.

d) En lesbio, $\alpha>0$ ante labial.

Por tanto, las reglas que se refieren al eolio y al arcado-chipriota son diferentes y el tratamiento de las sonantes no puede considerarse una isoglosa que sirva para agrupar dialectos ${ }^{2}$.

6.ro. Caso particular es el de Strunk, quien en un artículo reciente ${ }^{3}$ ha tratado de explicar, a partir de una secuencia lateral más laringal * $H_{1}$,

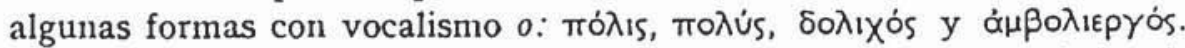
Es evidente, no obstante, que por sí sola esta secuencia no es determinante del vocalismo $o$. Dos ejemplos evidentísimos en contra son $\beta \dot{\alpha} \lambda \lambda \omega$

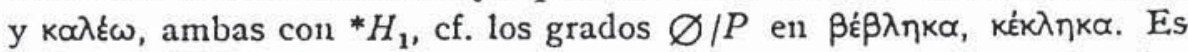
por ello preferible ver en mónis y moגús influencia de la labial anterior

1 La diferencia fundaruental consiste en que Wyatt cree que $\alpha p>$ op y Adrados cree que la influencia se produce sobre la vocal de apoyo antes de fonologizarse. Cf. también R. F. Adrados, Nicénico, dialectos paramicénicos y aqueo épicon, INM:RuTA 44, I976, pp. 88-9o.

2 El artículo es dogmático y en exceso simplificador, pero de él pueden sacarse ciertas verdades de interés: la existencia de factores fonéticos en ciertos resultados tildados de préstamos, la analogia de los grados plenos como factor influyente, el haber puesto de manifiesto que la visión tradicional del reparto a/o es en exceso simplista, así como la escasa fiabilidad de los tratamientos de $r$, ctcétera, para la dialectologia.

- K. Strumk, "Verkannte Spuren eines weiteren 'Tiefstufentyps im Griechischent, Glotta 47, 1969, pp. 1-8. La hipótesis remonta a Saussure, aunque Strunk no lo cita, cf. J. Gil, art. cit., p. 108. 


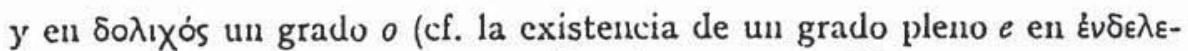

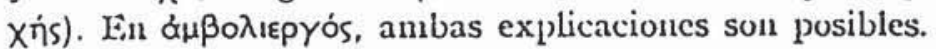

6.11. Un importante trabajo sobre el tema, tanto por su abundante pertrecho de materiales como por su afinadisimo análisis filológico de los mismos, pero especialnente por su honzadez metodológica, es un extenso artículo de If. Bader ${ }^{1}$ en el que examina unos materiales no puestos antes en circulación para el tema: los primeros miembros de compuesto, terreno bien conocido por la autora, que ha consagrado al mismo varios $y$ meritorios trabajos. Del examen de cstos datos, en contraposición con las vocalizaciones en la flexión, concluye esta profesora en primer lugar que los temas en vibrante $y$ nasal evolucionan

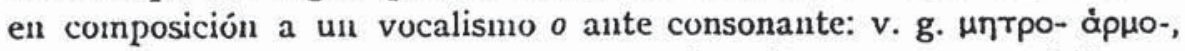
áleıpo-. En segundo lugar, que esta situación aparece también en palabras aisladas, como Opóvos, Kpóvos, etc. $\mathrm{X}$ en tercer lugar, que como contrapartida, hay categorías enteras que sólo tieuen $a$, como los acusativos plurales en - $\alpha$, los nombres en - $\mu \alpha$, etc., todo to cual testimonia la existencia posible en el primer milenio, como ya en micénico, de dos tratanientos de las souantes vocálicas: $o$ y $a$, en un mismo estado de lengua, una misma categoría morfológica o un mismo término, presentándose incluso ejemplos de vacilaciones en una misma inscripción. $\mathrm{Y}$ todo ello sin que sean imputables a orígenes dialectales diferentes, con lo cual niega la opinión de Cowgill ${ }^{2}$, que pretende ver una diferencia radical entre la situación del micénico y la de los dialectos del primer milenio.

No obstante, las consecuencias que de ello extrae no son de gran alcance. En fouética, se mantiene exactamente en el mismo punto de perplejidad en el que había quedado Meillet. Ein dialectología, observa que se trata de una normalización de grado más que de naturaleza, por lo que la vocalización de las sonantes no es un criterio determinante para clasificar los dialectos griegos.

6.I2. Hay que aludir, por último, a un artículo de Moralejo ${ }^{3}$ en el que se plantea la relación de los tratamientos del micénico con los

1 I. Bader, art. cit.

2 W. Cowgill, Ancient Greek Dialectology in the Light of Mycenaean, Ancient Indo-European Dialects (ed. H. Birnbaum y J. Pulnvel), Berkeley y Los Angeles, 1966, pp. 77-95.

3 J. J. Moralejo Alvarez, sSonantes y griego micénico", LMEERTA 4I, 1973. pp. $4 \circ 9-426$. 
de los dialectos del primer milenio para evidenciar que tanto $a$ como $o$ eran resultados alternativos de la vocalización de las sonantes que sólo posteriormente fueron regularizados en los dialectos, por lo que no pucde citarse ningún dialecto (lel primer milenio como descendiente del micéuico.

\section{CONCLUSIONES SOBRE t.A VOCALIzACión $a / o$}

7.x. Haciendo balance de to dicho sobre los vocalismos $a / 0$, cabe plantearse si podemos situar en un esquema coherente y cientificamente válido la multiplicidad de hechos observados y salvar las perplejidades que por ejemplo suscita en F. Bader la variedad de comportamientos que muestra el material exhaustivamente analizado por ella misma. La realidad de los hechos nos obliga a explicarlos, no a forzarlos desde perspectivas preconcebidas. A este respecto, creo que hay dos vías de aproximación al problema, una, que afecta a la metodologia en general, y otra, que se refiere al aspecto fonológico de la cuestión.

7.2. Respecto a esta primera vía hay que poner de manifiesto que laay una base metoclológica seguida sin crítica por casi todos los trabajos que he reseñado ${ }^{1}$ que consiste en el malentendido de que el métoclo linguístico (esto es, científico), debe coincidir con la regularidad simplista, aunque sólo sirva para una parte de los materiales, mientras que el aceptar la complejidad y la interdependencia de factores múltiples que intervienen en los procesos lingüisticos constituye, paradójicamente, la negación de la lingüística como ciencia. En efecto, se admite bajo estos presupuestos la existencia de dos resultados diferentes de un mismo fonema en uno de los casos siguientes:

7.2.1. Si en su coexistencia se pueden determinar las condiciones, esto es, si aparecen en entornos diferentes (no, en cambio, si aparecen en un mismo entorno).

7.2.2. Sí se pueden señalar niveles cronológicos o dialectales (considerándose "dialectales" como aproximadaniente coincidente con "geográficos»), en cuyo caso un resultado es fonético y el otro, préstamo.

7.2.3. Si uno de los resultados es el normal y el otro se debe a pre-

' lixcepeiones son los trabajos citados de Adratos, Bader y Moralejo. 
siones de tipo morfológico. Ėntonces, uno se considera fonético, y el otro, analógico.

7.3. Lo cierto es que los hechos no son tan simples como el anterior esquema invita a pensar.

7.3.I. Ein primer lugar, la dificultad de deternninar lo que constituye un "contexto igual" fue señalada ya en el siglo pasado por lingüistas de la talla de Schuchardt, Jespersen y Koch, quien en $I 896$ señalaba que el requisito del contexto idéntico nunca se cumplia. Sobre un fonema influyen no sólo los contiguos, sino también los que aparecen a distancia en la cadena hablada, a más de hechos de silabación, tempo, expresividad, valores estilísticos o incluso la pertenencia social del hablante, así como las influencias de la primera articulación sobre la segunda. Teóricamente, la posibilidad de estas influencias múltiples se admite, pero en la práctica, se opera como si los fenómenos que intervinieran en un proceso fueran simples y unilaterales; en otras palabras, se opera sólo con una parte de los factores en juego y se trata de explicar sólo con ellos la totalidad del hecho en cuestión.

7.3.2. En cuanto a las diferencias dialectales, la tendencia es a confundir dialectología con geografía o dialecto con compartimento estanco. Hay una dialectalización no geográfica, de índole social, en la que es posible que coexistan largo tiempo pronunciaciones diferentes. Naturalmente que esta dialectalización no geográfica nos resulta inaccesible, a la distancia temporal a la que nos hallamos de los hechos, pero no podemos negarla por el simple hecho de que la ignoramos. La dualidad de pronunciaciones es perfectamente pensable durante un largo tiempo y sólo secundariamente los dialectos impusieron (y nunca del todo) una solución u otra. Así pues es evidente que no se puede dar a las sonantes un valor para la distinción de dialectos más que concibiendo el predominio de una de las soluciones como resultado de una elección (prescindiendo de $u, i$, que no han sido elegidas por ningún dialecto y presentan el aspecto de soluciones residuales). Una de las soluciones cristaliza en una mayor frecuencia estadística ( $o$ en eolio, $a$ en dórico, jónico-ático, más repartidas en arcado-chipriota y micénico), pero entiéndase bien que no son tratamientos exclusivos ni inclependientes de uno u otro dialecto, sino hechos de elección y generalización en los que quedan en mayor o menor medida huellas de la situación antigua. A la vista de los datos en el tema que nos ocupa, creo que es claro que es ésta la conclusión que hay que obtener, de acuerdo además con 
ideas sostenidas por Bartoněk ${ }^{1}$ respecto a la coexistencia de dos variantes lingüísticas en un misıno estado de lengua.

7.3.3. En cuanto a la analogia, hay que señalar que no siempre es un fenóneno producido a posleriori, sino que el cambio fonético puede ser encauzado desde la primera articulación, conı veremos ell § 7.7.6.

7.4. Ante la existencia de estas limitaciones y terrenos no accesibles a la metodología al uso, creo que resulta extremadanente inapropiado un dogmatismo a ultranza que decida, sin basarse en los hechos, que un tratamiento es único y exclusivo de un dialecto y cuando aparece otro se debe a razones no fonéticas (préstamos, analogías, etc.).

7.5. Pero hay algo mucho más importante: pienso que hay un error de base en las interpretaciones que frecuentemente se hacen de este cambio desde un punto de vista fonológico. La vocal de apoyo, causante de la posterior aparición de vocales en la vecindad de las antiguas sonantes vocálicas en grado cero, no es un fonema, sino, en la precisa terminología de Hamp ${ }^{2}$ un vocoide alofónico. Fillo comporta una serie de consecuencias: a) que el vocoide alofónico no está situado en un haz de correlación; b) que no posee rasgos distintivos frente a las demás vocales; c) que, por lo tanto, no está defendido por oposiciones que puedan provocar, en sus alteraciones, los fenómenos bien conocidos ya desde las importantes aportaciones de Mrartinet al tema, de tracción, propulsión, etc. La invasión del margen de seguridad de una vocal por una realización de la vocal de apoyo no provocaba conflictos distintivos por el mero hecho de que la vocal de apoyo no poseía de antemano rasgos distintivos; d) por todo lo dicho, los cambios que se refieren a las sonantes vocálicas indoeuropeas no pertenecen ni a los llamados cambios por alófonos ni a los llamados cambios por fonemas. Se trata más bien de uno de esos cambios que suelen denominarse "esporádicos" $y$ tiene su origen en una tendencia a una silaba fonológicamente normal. La ley fonética que se refiere a las sonantes no puede enunciarse diciendo que ${ }^{*} \gamma$ da $\alpha \rho$, etc. como frecuentemente se hace, sino más exactamente diciendo que una sílaba TRT se resuelve en una silaba

1 A. Bartonék, aMycenaean Koine Reconsidered», Proceedings of the Cambridge Colloquium on Mycenaean Studies (ed. L. R. Palmer y J. Chadwick). Canbridge, ig66, p. 97.

2 E. P. Hamp, art. cit. 
con vocal 'TER'T o TRL'T y que una sílaba TRL puede (no siempre) resolverse en TERI:. A partir de todo cllo, el proceso puede describirse como siguc:

7.6. En términos fonolugicos, lo que se ha prodlucido es una fonologización del vocoide alofónico que acompañaba a las sonantes indoeuropeas, en una vocal plena. ¿De qué timbre? Descle luego, en el de una vocal ya existente en el sistema de la lengua, lo cual es lógico, porque no hay que mantener por transfonologización una distinción entre voca! plena y vocoide que fonológicamente no existía.

7.7. De otro lado, el resultado vocálico, fuera cual fuese, no chocaba con las normas de distribución fonológica: esto es, una solución up, por ejemplo, se tolera por la existencia de antiguas secuencias ${ }^{*} u+r$ $\left(0^{*} H^{* 0}+r\right)$, op, por los grados $o$, etc.

7.8. Sobre este vocoide alofónico en trance de fonologizarse se ejercen varias presiones de intensidad variable que pueden sintetizarse como las siguientes:

7.8.r. E.l carácter de las sonantes, sonoras y abiertas, que provoca una tendencia no condicionada contextualmente a desarrollar un apoyo vocálico de abertura máxima: $a$. De ahí que el tratamiento óp- en inicial sea absolutamente mayoritario y panhelénico. Eil contexto (consonante final de la palabra anterior) varía, y se impone el tratamiento 110 condicionado.

7.8.2. Si la sonante está palatalizada, la tendencia es a una vocal de apoyo de timbre palatal. Es el caso de $i$ en oíripw, de $c$ en la palatalización secundaria del lesbio, Пє́pparos, etc.

7.8.3. Influencia de la consonante vecina. Así, la labiovelar influye en un timbre $u$, la labial y gutural, en $u$ o en $o$, etc.

7.8.4. Influencia de fonemas a distancia, estudiados para la vocalización $u \mathrm{y}$ para algunos casos de 0 .

7.8.5. IFactores expresivos, cuya intervención pudo observarse asimismo especialmente en el caso de la vocalización $u$.

7.8.6. Influencia de la primera articulación, que puede encauzar el cambio fonético de diferentes formas: 
a) La pertenencia de la apalabra afectada por el cambio a una seric morfológica. Así se explican el timbre o analógico de grados plenos con o larga, olsservadia por Wyatt (épotós, бтрото́s) o la consideración de una $o$ producto de grado cero como un grado $o$, tipo $\theta$ póvos. Por influjo morfológico de los verbos reduplicaclos en $-\mu$ se explicaba la aparición de $i$ en raíces que contienen una sonante en grado cero. Aún puede añadirse el caso de las series de numerales en las que se han operado regularizaciones secundarias de una u otra vocalización: тв́тортоS junto a ठ́́kotos, etc.

b) Otra forma de actuación de la morfología es la tendencia a regularizar los resultados cuando la sonante en cuestión se halla en una desinencia: de ahí que el acusativo plural en ${ }^{*}$-ns se resuelva por -as en todos los dialectos, aunque fonéticamente podía tender a realizarse con vocales diferentes. Asimismo para conservar la unidad de la categoría morfológica, el sufijo ${ }^{*}-m e n /-m o n$ en grado cero se resuelve siempre ell $-\mu \alpha$ en los sustantivos, pero en - $\mu$ o en los compuestos. Tal regularización no sólo produce en los morfenuas, sino también en frontera de morfema (hay siempre $\alpha$ en los dativos plurales: marpóal, etc.,

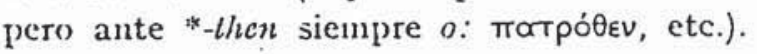

c) En otros casos se trata de la intervención de la serie léxica a la que pertenece una palabra: la serie ya analizada de vocalizaciones $u$ de sonantes en grado cero en palabras de origen microasiático puede ser un buen ejemplo de ello. Lis evidente que no hay que pensar que fueran prestadas ya con una $u$ que no tenían en las lenguas de origen, sino prestadas con sonante en grado cero y que evolucionaron a $u$ por su carácter "exótico". Igual hay que decir de cierto léxico uinternacional" del que sería un buen ejemplo la palabra de la 'dracma', resistente a presentar diferencias dialectales, etc.

7.9. Hasta aquí pienso que puede llegar por ahora el método lingïístico: a fijar las condiciones, los factores que intervienen en el cambio, qué resultados tienden a imponerse y qué dialectos tienden a generalizar una solución u otra. Los hechos son pues más complejos que la mayoria de los esquemas propuestos y opino que la estrechez de esquemas no hace avanzar la lingüística, sino que más bien la detiene, la empobrece $y$, lo que es peor, la falsea. 


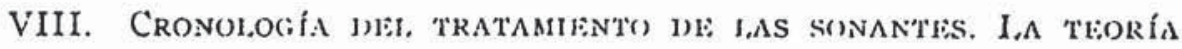
DE: Hrouli:CK

8.r. Q2ueda por viltimo aludir brevemente al problema de la cronología del tratamiento de las sonantes, concretamente a la hipótesis enunciada por Heubeck ${ }^{2}$ acerea de la conservación en nicénico, sin tratar, de la vibrante vocálica.

8.2. Las principales conclusiones de Heubeck son las siguientes:

8.2.r. La secuencia ${ }^{*}-\mathrm{rH}_{2}$ se había tratado ya en micénico y había evolucionado a $-r \bar{a}$, como se ve en casos como ka-ra-te-ra|krätēra|, para-ke-se-n |Präkscus|, ka-ra-a-pi |kraha( $p$ )phil, etc.

8.2.2. La sonante ${ }^{*} \gamma$ está aún sin tratar y se escribe con dos variantes Co-ro/Co. E1 autor interpreta como meranente gráficas la vacilación entre ma-lo-pu-ro y ma-to-ro-pu-ro por estimar que es imposible que un mismo lugar tenga nombres diferentes.

8.2.3. Apoya su interpretación de los hechos la existencia de es-

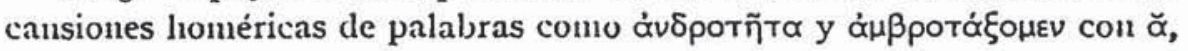
que presuponen, en su teoría, antl-|, |amtl-|.

8.3. La hipótesis de Heubeck, aunque sugestiva, se ha visto sin embargo contestada por una serie de argumentos:

8.3.r. Así Moralejo ${ }^{2}$ considera como regla central de la ortografía micénica la presentación clara del centro silábico, de modo que una grafía como 10 - para $\left|t_{r}\right|$ lo desdibujaría. Ein cuanto a las escansiones homéricas, propone como solución asignarles una fecha premicénica, no excesivamente alta, aunque en algunos casos la irregularidad mé-

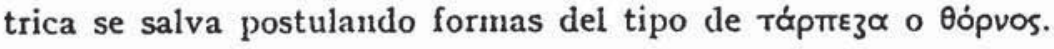

8.3.2. Por su parte Adrados ${ }^{3}$ critica también la validez de los casos de correplio para extraer conclusiones sobre el tratamiento de las sonantes, ya que existen otras fórmulas también en Homero en las que se requiere la vocalización de las sonantes. Además considera increible

1 A. Heubeck, art. cit.

2 J. J. Moralejo Álvarez, art. cit. p. 422 y ss.

3 F. R. Adrados, Micénico..... 
una vocalización de las dos líquidas seguidas y más increíble aún que en IIomero estuviese vocalizada la primera y no la segunda. A ello lıay que unir el hecho de que la $d$ epentética está ya documentada en

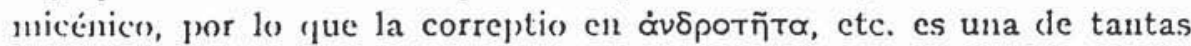
licencias de carácter métrico. De otro lado sigue a Moralejo para poner de manifiesto que junto a los casos con grafía $o$ existen otros con grafía $a$ y las coincidencias entre los resultados micénicos y los de los dialectos no pueden deberse al az.ar.

8.4. Además de los argumentos reseñados creo que existen otros de diferentes índoles que dificultan gravemente la aceptación de la hipótesis de Heubeck.

8.4.I. El primero se refiere a la imposibilidad de que se designe por dos nombres un mismo lugar, argumento fundamental de Heubeck ante la presencia de alternativas como ma-lo-pu-ro/ma-to-ro-pu-ro. No me parece imposible que sucediera en Pilo lo que sucede en otros lugares en los que aparecen designaciones vacilantes de un mismo lugar o de un epíteto divino; como en latín: en la Sententia Minuciorum ${ }^{1}$ el mismo tío se llama tres veces l'rocobera y tres l'orcohera, una vaci-

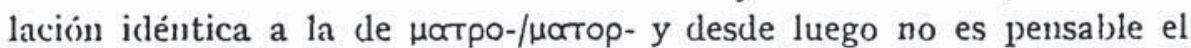
mantenimiento $\mathrm{lle}^{*} \boldsymbol{r}$ en latín en el Ir 7 a. C. para justificarla. En griego

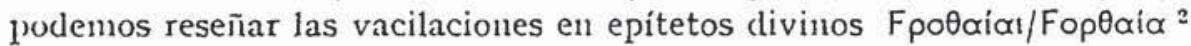

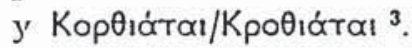

8.4.2. Ya hemos visto como resultaba poco verosimil que las coincirlencias en las grafías $a$ y o cntre el micénico y los dialectos del primer milenio se debieran al azar. I,a inverosimilitud se agrava si a estas coincidencias añadimos las que se producen en los casos cle vocalización $u$.

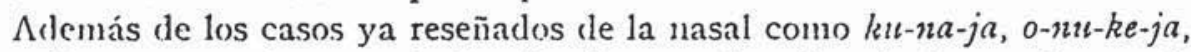
podemos presentar para la líquida algunos ejemplos: $p o-p u-r \mathrm{O}_{2} \mathrm{cf}$. mopqúpєos; ku-rc-we, sea que se interprete como /skiulewes/ 'expoliadores' o) como /shulerves/ 'vestidos de cuero', se presupone un $\sigma k u ́ \lambda \lambda \omega$; ku-ro

1 CIL $1^{1} 199=I^{2} 5^{84}=\mathrm{V} 7749$.

2 I:. Schwyzer, Dialeclorum Graecarsm exempla epigraphica poliora, Leipzig, 11)23. nimeros 5.1 y 5.2 .

3 Ibid'm, numeros 67.3 y 67.5. I.os ejemplos, por supuesto, pueden ampliarse. Cr. la vacilación en $\mathrm{cl}$ nombre de un misno monte en el texto hetita de Ihiyanka, K Bo III 7 II: cn linea 2 I se denomina Zaliyanu y en la 25. Zalinu, o las múltiples variaciones en los topónimos eu español, como Elsonza/Ilsonza/Eslonza/Elisonzal Flisontie/Alisonza, cf. R. Menéndez Pidal, Origenes del Español, Madrid, 19;2 1. 311 . 
que Ruijgh 1 interpreta como |K̈ulyos/ 'bebedor', como resultado del grado cero de la raíz. *skel-; ku-sa-me-ni-jo interpretado por Ruijgh 2

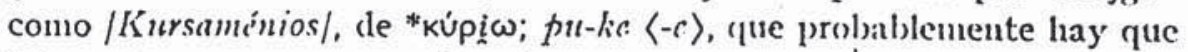
interpretar como un caso de múpyos.

Estas coincidencias, además de resultar increfbles si no se piensa que las sonantes estaban ya tratadas en micénico, nos obligarian a aceptar para ${ }^{*} r$ cinco grafías: Co (ma-to-pu-ro), Co-ro (ma-lo-ro-pu-ro), $\mathrm{Ca}(k a-p o),-r a-(e-r a-p e-m e-n a)$ y $C u\left(p o-p u-r o_{2}\right)$, lo que resulta aún más inrrefble.

8.4.3. Pero hay aún dos cuestiones de cronología relativa respecto al tratamiento de las sonantes que es preciso reseñar. La prinera de ellas es que si, como señala Heubeck y resulta evidente, ya se había tratado la secuencia ${ }^{*} r \mathrm{H}_{2}$ en $r \bar{a}$, cabe preguntarse cuál es el mecanismo de ese tratamiento. La explicación más verosímil del mismo ${ }^{3}$ es que la $\bar{u}$ procede de un alargamiento compensatorio a partir de una doble vocalización: ${ }^{* \circ} r^{\circ} H>r \bar{a}$, fenómeno que presenta paralelos en eslavo. Ello quiere decir que ${ }^{*} r$ recibió la vocal cle apoyo antes de la caída de ${ }^{*} H$. Si no, el tratamiento de ${ }^{*} r H$ no se distinguiría del de la sonante simple. Lo mismo hay que decir del trataniento propuesto por Strunk al que ya hemos aludido y que ya es micénico, como se ve por po-to-ri-jo, po-to-ri-ke-ta, po-ri-po-de, do-ri-ka-o, etc.

8.4.4. La segunda cuestión de cronología relativa es que la ${ }^{*} r$ tuvo que vocalizar antes del tratamiento de $* r i$, , como lo evidencian dobletes

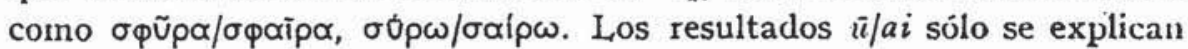
a partir de dos vocalizaciones con breve, $u / a$, pasadas a $u / a i$ cuando se consuma el tratamiento del grupo $r i \underline{\text {. }}$ Si, como quiere Ruipérez ${ }^{4}$ tal tratamiento está ya consumado en micénico, la vocalización de ${ }^{*} r$ tiene forzosamente que ser anterior, esto es, premicénica. De haberse producido vocal de apoyo en el estadio en el que la sonante estaba palatalizada por la $i$ (o en la hipótesis de Ruipérez, en el estadio palatal geminado), la vocal sería la $i$.

Así pues, tanto la pérdicla de las laringales en las series ${ }^{*} r H_{2},{ }^{*} l H_{2}$ como el tratamiento del grupo ${ }^{*} r \underline{i}$ son posteriores a la vocalización de las sonantes en griego.

\section{Alberto Bernabí Pajares}

2 C. J. Ruijgh, ob. cil., p. 175, n. 388.

2 Ibidem, p. 143.

3 Cf. F. R. Adrados, Estudios..., p. 195 y ss.

- M. S. Ruipérez, "Le dialecte mycénien», Acta M\%̈cenaea, Salamanca, 1972

I, pp. 136-166. 